\title{
Analysis and Issues in Cloud Operating System
}

\author{
Debasree Basu, Indusai G, J.Vijayashree, J.Jayashree, \\ *Ronnie D. Caytiles and N.Ch.S.N Iyengar \\ School of Computer Engineering, VIT University, \\ *Hannam University, Korea \\ Vellore - 632014, Tamil Nadu, India \\ nchsniyr@vit.ac.in
}

\begin{abstract}
The conventional operating system usually works on a particular workstation but the same when implemented on a distributed environment like cloud faces many problems. This paper analyzes and explains how does the cloud operating system works in order to resolve these problems. It also differentiates the working mechanism of both based on basic storage management service provided by the cloud. At the end it provides the issues and the future challenges faced in the cloud operating system.
\end{abstract}

Keywords: distributed operating system, storage management

\section{Introduction}

The operating system is most important software running on a computer. It provides an interface between the user and the hardware. The operating system is responsible for all major functionalities in the computer. The basic functionalities of the operating system are resource management, process scheduling and management, event handling. It also allows users and other software's running on top to communicate with hardware. It provides I/O management.

There are basic two paradigms for designing an operating system. One is message based and the other is object based. In the message based operating system [1] the interprocess communication takes place by message passing. Each of the nodes has a message passing kernel. It supports both local and global communication. In local communication the messages are passed to the processes in the same node while in the global communication the messages are passed to the processes at other node. The message passing sometimes is achieved with the help of network manager. In message passing system, access to the system services is by message passing in contrast to that of UNIX where the system services can be accessed only with the help of protected procedure calls. ${ }^{1}$

In the object based operating system [1], the services and resources are combined together to form a single entity called class. Objects are instances of these classes. The object consists of the data and set of operations to be performed on the data. These functions are called methods. The object based operating system has the advantages of the object oriented design. It provides portability; the objects can be easily transferred from one node to another. The objects also facilitate code sharing. In order to perform a particular function on a data we have to invoke the functions using the necessary objects. The code sharing can be easily achieved by two processes sharing the same object and the calling the functions. To ensure concurrent execution of processes in the object it must have a mechanism for semaphores, locks in order to maintain consistency and mutual exclusion if needed. In contrast to message based where the level of concurrency is

Ronnie D. Caytiles is the corresponding author. 
necessary to be determined in advance there is no such need in object based operating system as concurrency is achieved by concurrent execution of threads in the object. The main motive for object based system is functionality. The code is divided into classes and objects based on their particular functionality. The examples of object based operating systems are Genera, Chorus, NachOS, and PenPoint.

\section{Clouds Approach}

Cloud is a distributed operating system that integrates set of nodes into a centralized system. The cloud uses object based operating system paradigm. The Figure 1 shows the cloud object.

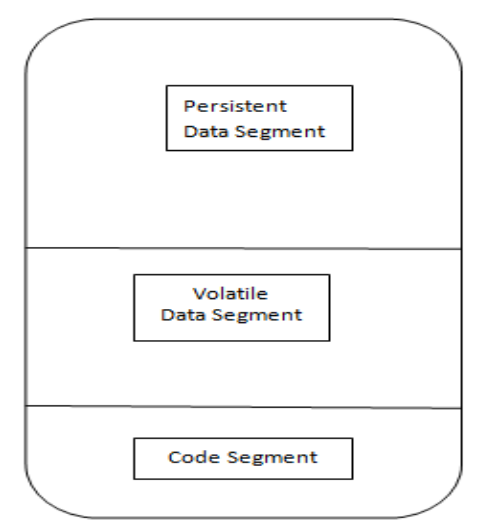

Figure 1. Cloud Object

The cloud object are very large grained and contain encapsulation of entire virtual address space. They store large amount of data and program because of the overhead associated with the invocation of the object. The cloud object has following characteristics.

\subsection{Segmented Address Space}

The cloud object contains entire virtual address space. These address space is segmented. As in Figure 1, the cloud object usually consists of three segments. The code segment contains user defined code. The persistent data segment contains the persistent data objects and the persistent heap which is used to store the temporary data which becomes the part of persistent object. The volatile data segment contains the memory for temporary storage allocation during the execution.

\subsection{Passive}

The cloud objects are passive. It does not contain process or any active entity. It mainly consists of the data and the methods to operate on that data. The clouds virtual address cannot be accessed from anywhere outside the object. The only way to access the address (data) is by the code inside the object.

\subsection{Persistent}

The clouds objects are persistent. They remain in the memory until they are explicitly deleted and are unaffected by any system crash or shut down.

\subsection{Object Invocation}

The cloud uses object thread mechanism. The only active entity in the object is the user thread. The thread executes the user program. It traverses all the objects in the code and 
invokes the necessary methods inside the objects. The different objects have different address space. Therefore clouds thread is not bound to single address space. The arguments in the methods needs strictly to be data, memory addresses cannot be passed as an argument to the methods. The addresses reference in one object may be invalid in the other object as they have different addresses spaces. For data sharing the cloud creates the object for the shared data and when the access to the shared data is needed simply the cloud object methods are invoked. The object may call other object in a cascading manner. The control flow is achieved by threads invoking objects while the data flow is achieved by the parameter passing in the methods.

\subsection{Recoverable}

The cloud objects are recoverable. It is possible to rollback back to the safe state in case of any abnormalities during the execution of the code.

\section{Problems in Conventional Operating Systems}

Cloud computing has now found its place in all branches. All applications are readily available on the clouds and almost all of the organizations have some part of their application currently running on cloud. Considering the conventional operating system like Mac, Windows, Symbian have many shortcomings. In this section we will discuss the general shortcomings of the conventional operating system and see how cloud can provide solution for these problems.

\subsection{Hardware Problem}

The hardware we buy usually gets outdated within a span of years. Due to continuous evolving technology we land with old systems that are unable to run latest software or applications. If it is a desktop system it is possible to upgrade the system, but this continuous upgrade of the system will become a costly affair. Also there is problem of compatibility; OS is designed to run on a specific hardware [6]. For example Mac OS can only run on Apple system and is not compatible with any other hardware.

This problem can be solved using cloud. In cloud the hardware is elastic. We can choose to configure our system based upon the requirements. It's also possible to upgrade to degrade our configuration with ease. There is no need to physically buy the components and upgrade them. In cloud the billing is based upon the use and therefore it is cost saving. Hence it is possible to get the speed and facilities of a superfast computer on our home outdate system using cloud.

\subsection{Transfer Data}

Many times we need to transfer data from one node to another. This transfer of data between the nodes is very slow. We can use two kind of medium for transfer of data [2]. Hardware like pen drive, DVD, hard drive etc. or Network like Bluetooth, WI-FI, Internet etc. The network transfer is usually faster than the hardware transfer and also reliable.

In cloud computing the transfer is through internet. Now suppose you want transfer a huge file from one system to another. Normally we will have to upload the file in the internet and then send it. But using cloud the content is already present in the network and we only have to send it. The person to whom we have to send the file can remotely access the file from the cloud.

\subsection{Accessibility}

In today's world accessibility is a big issue. People need to access the data remotely and also they want to access the data in different environment. People need easy and dynamic operating system which is not possible using conventional operating system. In 
cloud the user data is present in the data server. The data server holds persistent data and is unaffected by power cut or system failure. These data can be easily accesses remotely. Using cloud data can be accesses anywhere and in every known environment.

\subsection{Data Backup}

It is very important to take backup of your system data. In any case due to system failure there is chance of losing all your data. This backup process is quite time consuming. While in cloud the data is already present in persistent data servers. These data objects are unaffected by power cut or system failure. The data remains the server unless it is explicitly removed by the user.

\subsection{Power Consumption}

The common system consumes very high power. As the processor speeds increases the power consumption increases and so does the heat generated by the system. The system gets hot and it is essential to maintain the system in a cooler environment. Also in conventional system there is a problem of power failure resulting in loss of data. In cloud computing there is no fear for data loss due to power cut. Due to many advances in the cloud technology the power consumed by the cloud is quite low. According to recent study conducted by students from Lawrence Berkeley National Laboratory (Berkeley Lab) [14], it is shown that by moving 86 million of US office works to the cloud we can save up to $87 \%$ of IT energy. This much energy is enough to power the entire city of Los Angeles for 1 year.

\section{Difference between Conventional OS and Cloud OS}

\subsection{Storage Management and Storage Mapping}

In this section we have discussed the basic storage management and addresses resolution process in cloud and how it differs from the conventional operating system. In conventional operating system we have main memory and secondary memory. The main memory is considerable less in size as compared to that of secondary memory, which is ample in size. All the programs originally are stored on the secondary memory when a program is ready to run it is brought in main memory. The entire program is not brought initially, only a part of program is in main memory. The program is brought in main memory in parts called pages. As per the request the pages are swapped in and out of the main memory. The user is unaware of this process and assumes that the entire program is in the main memory. This creates an illusion that the memory available for the processor is more than is main memory. All the addresses generated by the program are resolved in main memory, but due to this swapping mechanism and demand paging it seems that the address available for the user is larger than its main memory. This address space is called virtual memory. The Figure 2 below shows the basic address resolution between the main memory and virtual memory. 


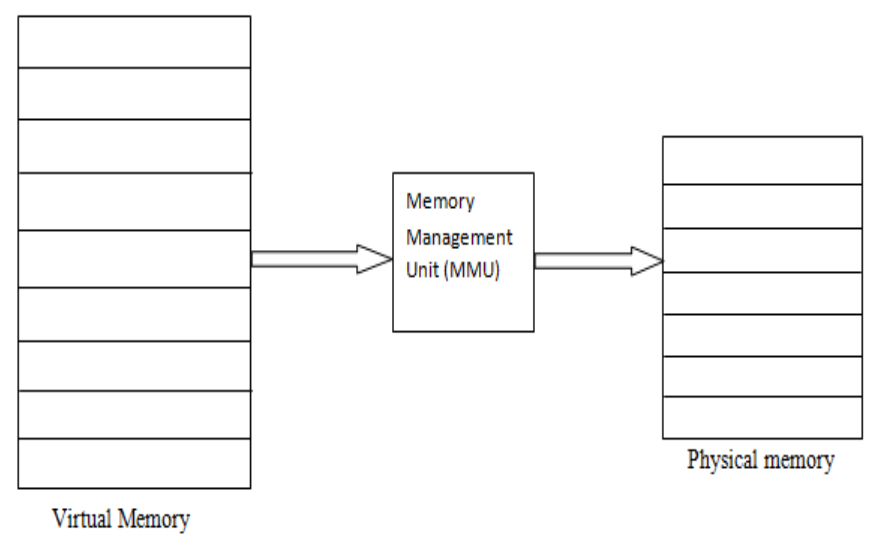

\section{Figure2. Address Resolution between VM and MM in Conventional OS}

The virtual memory is made up of pages and the physical memory is made of frames. The page size is same as that of the frame size. When the system generates an address it is virtual address and refers to virtual memory. The address is of form page number and offset. Using the page number we look up into the page table, which has the frame number in which the page is placed. If the page is not in main memory then it is brought in main memory by swapping and an entry is made in the page table. After getting the frame number the virtual address is converted into physical address.

In case of cloud, there are three types of machines or servers. The user workstation is the system present at the user end. It actually provides the interface for the user to write and execute the program using the cloud with the help of data server and computation server. The computation server is the server where all the necessary computation takes place. The computation server need not have any memory. All the objects are present in the data servers. The computation server may also have some memory and can serve as both data server and computation server. Whenever the user wants to run the program, user thread is created. The cloud then has to decide on which computation server the thread must execute. This depends upon various factors like the load present at the server, the network traffic, the presence of necessary cloud objects on the nearby servers. Figure 3 shows the basic operation of the computation server. The cloud object uses segmented memory. The object contains entire virtual address space. The data and the related code are encapsulated together to form a template. The template also contains information on how object memory is to be created. It contains information on how the segments must be mapped to create object memory. This is called as the object description. Each computing server has Active Object Table (AOT) and Active Object descriptors (AOD) which lists all the active objects in the server and their mapping Description. The AOD contains the mapping information for the object. In Figure $3, t$ is the thread invocation for execution by the user. Whenever the cloud object is needed the thread searches for the object using local thread manager. The local thread manager first searches for the object in the AOT present in the server. If there is entry for the object in AOT, means that the object is active. It gets the descriptor for the object form AOD. If it is not present in the AOT it gets the object from the other servers, usually data server using Remote Procedure Call (RPC). It can search for the object using any appropriate, efficient searching algorithm. It gets the object and its descriptor for the object template and makes and entry for the object in AOT and a corresponding entry in AOD.

The mapping of object into the computation server takes place two fold. First is the mapping of segments to object memory windows. This is mapping between object and the virtual memory of the computation server and the other is mapping of segments to the physical memory. This is the mapping between virtual memory and the physical memory. The object descriptor gives information about the mapping of object windows and the 
memory segments. Each computation server consists of Active Segment Table (AST) and Active Segment Descriptors (ASD). This together forms the virtual memory. Each object has an entire address space associated with it. This address space is further segmented. The computation server has to associate appropriate segments with this object window. When a segment is associated with a object then the segment is said to be activated.

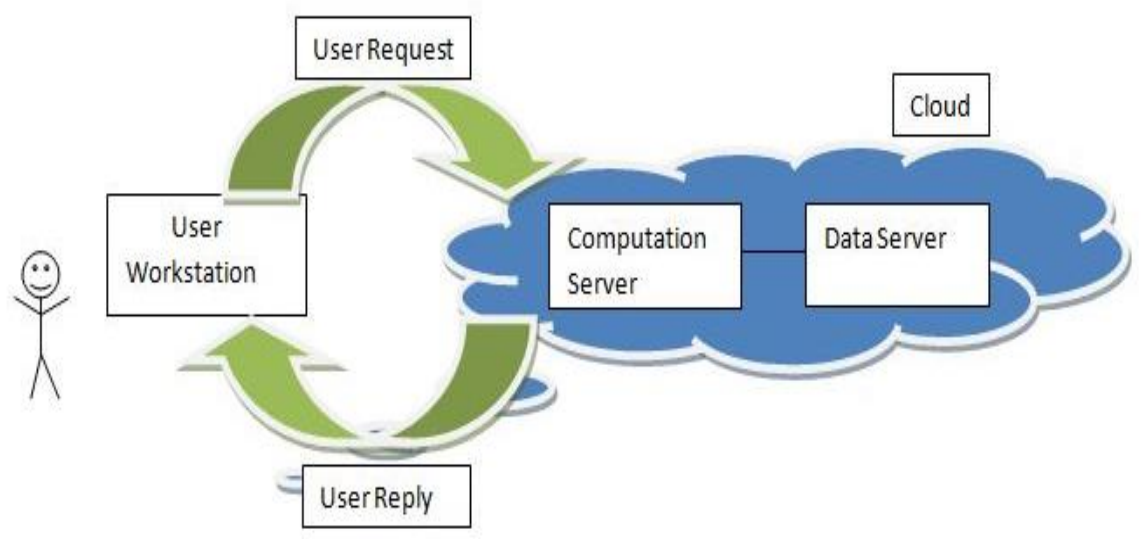

Figure 3. System Architecture

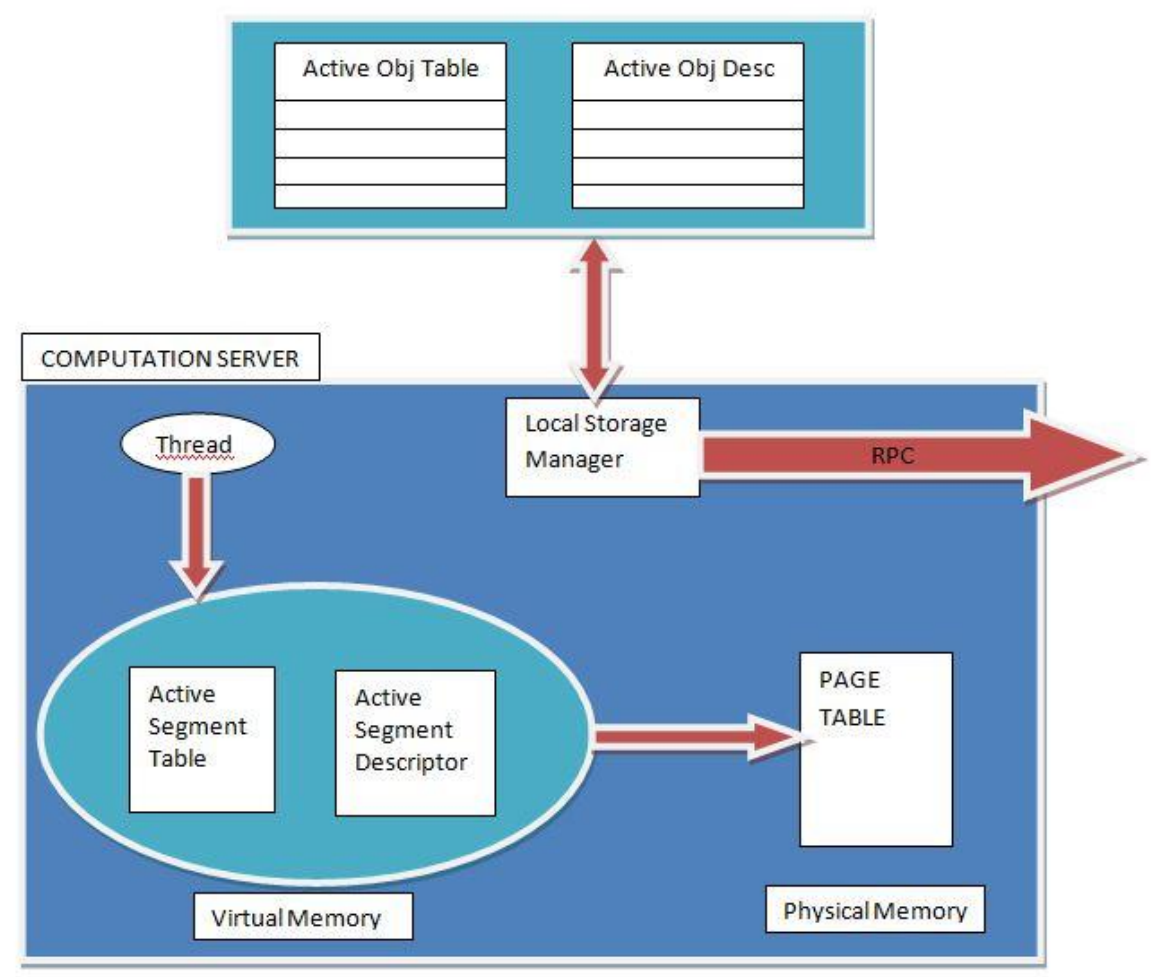

Figure 4. Functional View of Computational Server

The AST contains the list of all the active segments in the server and ASD contains the description regarding the object to which the segment which indicates. The segments are internally organized in the form of tree where the root node is segment descriptors; it contains the metadata regarding the segments as to the number of segments available. The active segments are present at the leaf of this tree, whereas the path from the root to the leaf indicates the object which occupies the segment. On receiving a new object the server searches for the appropriate segments and maps them to the object window and creates 
and entry in the AST and ASD. The mapping between the active segment and main memory, which is organized in form of pages, is by using Page Table (PT). The page table has a entry for each page or frame of the main memory. The segments of the virtual memory are internally fragmented into pages of same size as that of main memory page size. These pages are brought into main memory dynamically based on the need. This mechanism is called dynamic paging. The pages are moved in and out of the main memory and this deactivation depends type of object segment the page holds i.e. volatile or persistent. Various page replacement algorithms can be used for demand paging mechanism.

\section{Conclusions}

In this paper we have given a generalized idea about the cloud operating system. We have specified how cloud provides a solution to certain problems that prevails in the conventional OS. We have discussed how the cloud provides the storage and does the address mapping while execution. This paper can be useful for any those who wants to proceed with any changes in the memory management done at the computation server and improve the address mapping making it more fast and efficient.

\section{References}

[1] Partha Dasgupta, Richard J. LeBlance et. Al. , "The clouds distributed operating system"

[2] A. S. M. Abdul Kader "CLOUD COMPUTING: THE BASE OF FUTURE OPERATING SYSTEMS" International Journal of Scientific \& Engineering Research, Volume 4, Issue 1, January-2013 (ISSN 2229-5518).

[3] Fabio Pianese Peter Bosch ,"Toward a Cloud Operating System”, Alcatel-Lucent Bell Labs.

[4] David V. Pitts Partha Dasgupta, "Object Memory and Storage Management in the Clouds Kernel".

[5] Michael Armbrust, et.al., "A view of cloud computing", Communications of the ACM, vol. 53 no. 4. April

[6] Omesh Kumar, Deepak Rai, “ Cloud as an Evolutionary Operating System”, Special Issue of International Journal of Computer Applications (0975 - 8887) on Issues and Challenges in Networking, Intelligence and Computing Technologies - ICNICT 2012, November 2012.

[7] Sathis Menon, Parha Dasgupta ,"Asynchronous Event Handling in Distributed Object-Based Systems”.

[8] Judith Hurwitz " "The Role of the Operating System in Cloud Environment", A Hurwitz White Paper.

[9] Michael Armbrust, Armando Fox "“A view of Cloud Computing”.

[10] Sathis Menon, Parha Dasgupta ,"Asynchronous Event Handling in Distributed Object-Based Systems".

[11] "Effective storage managementand data protection for cloudcomputing", IBMSoftwareThought Leadership White Paper,December 2011

[12] Mrs.S.Selvarani, Dr.G.Sudha Sadhasivam "Improved Cost based Algorithm for Task Scheduling in Cloud Computing.

[13] VMWARE white paper .

\section{Authors}

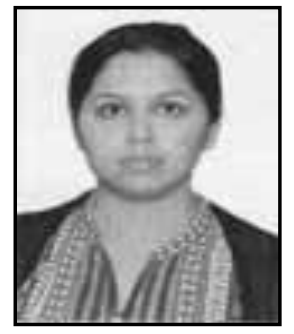

Debasree Basu is pursuing M.ScComputer Science at School of Computing Science and Engineering, VIT University, Vellore. Her area of interests are Algorithm design an Pattern Recognition ,operating Systems and cloud computing

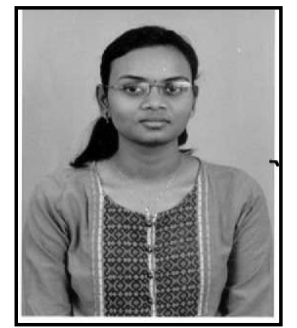

Indusai $\mathbf{G}$ is pursuing M.ScComputer Science at School of Computing Science and Engineering, VIT University, Vellore. Her area of interests are Software Engineering,operating Systems and cloud computing. 

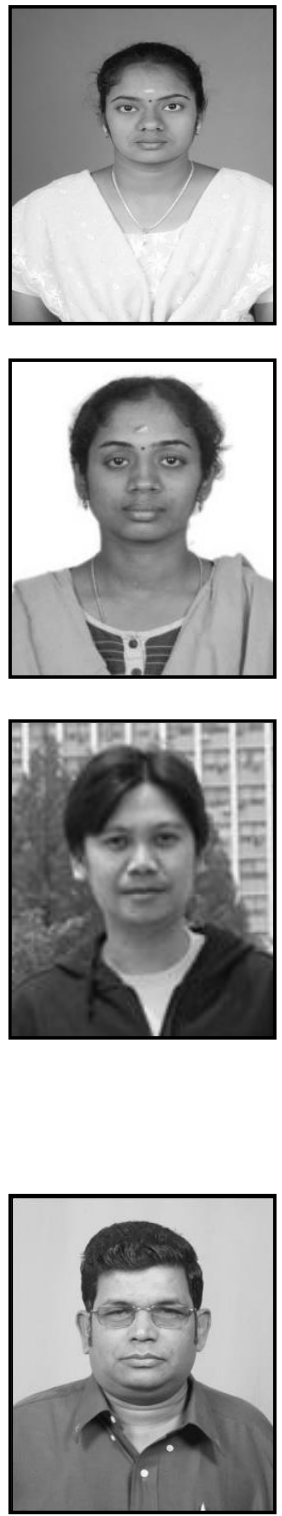

Ronnie D. Caytiles, He had his Bachelor of Science in Computer Engineering- Western Institute of Technology, Iloilo City, Philippines, and Master of Science in Computer Science- Central Philippine University, Iloilo City, Philippines. He finished his Ph.D. in Multimedia Engineering, Hannam University, Daejeon, Korea. Currently, he serves as an Assistant Professor at Multimedia Engineering department, Hannam University, Daejeon, Korea. His research interests include Mobile Computing, Multimedia Communication, Information Technology Security, Ubiquitous Computing, Control and Automation.

N.Ch.S.N. Iyengar (b 1961) currently Senior Professor at the School of Computer Science and Engineering at VIT University, Vellore-632014, Tamil Nadu, India. His research interests include Agent-Based Distributed Computing, Intelligent Computing, Network Security, Secured Cloud Computing and Fluid Mechanics. He had 30+ years of experience in teaching and research, guided many scholars, has authored several textbooks and had nearly 200+ research publications in reputed peer reviewed international journals. $\mathrm{He}$ served as PCM/reviewer/keynotespeaker/Invited speaker for many conferences. He serves as editorial board member for many international journals, reviews papers for many conferences with an interest of serving to the education community. 